\title{
Enteroparasitoses em manipuladores de alimentos de escolas públicas em Uberlândia (Minas Gerais), Brasil
}

\author{
Carlos Henrique A. de Rezende, ${ }^{1}$ Julia Maria Costa-Cruz ${ }^{1}$ e \\ Margareth L. Gennari-Cardoso ${ }^{1}$
}

RESUMO Procedeu-se ao controle das enteroparasitoses em manipuladores de alimentos de escolas públicas de primeiro grau de Uberlândia, Minas Gerais, a partir de estudo prospectivo baseado em inquérito parasitológico. Foram acompanhados 264 indivíduos de 57 escolas, com três coletas de fezes (C), sendo C1 em setembro de 1989, C2 em abril de 1990 e C3 em setembro de 1990. Entre os participantes 259 eram do sexo feminino e cinco do sexo masculino, com idades entre 20 e 66 anos. As 792 amostras de fezes foram conservadas em formol a 10\% e analisadas pelo método de Hoffman, Pons e Janer. A ocorrência de parasitas intestinais foi de 17, 10 e 10\% para C1, C2, e C3, respectivamente. Em C1 detectou-se: Giardia lamblia (8\%), ancilostomídeos $(6 \%)$, Ascaris lumbricoides (3\%), Entamoeba histolytica, $(2 \%)$, Strongyloides stercoralis, Hymenolepis nana, Taenia sp. e Trichuris trichiura (menos de 1\% cada um deles). Em C2 detectou-se: ancilostomídeos (5\%), G. lamblia (2\%), A. lumbricoides (1\%), S. stercoralis, H. nana, Taenia sp., Enterobius vermicularis (menos de 1\% cada um deles). Em C3 observou-se: ancilostomídeos (5\%), G. lamblia (3\%), A. lumbricoides (2\%), S. stercoralis $e$ E. vermicularis (menos de 1\% cada um deles). Os indivíduos infectados receberam tratamento específico após o diagnóstico em cada coleta. Recomendamos a implantação de vigilância epidemiológica através do exame parasitológico periódico, tratamento específico e treinamento sobre manejo higiênico de alimentos e higiene pessoal para todos os manipuladores de alimentos.

A magnitude global e a importância das infecções parasitárias e das possíveis medidas para seu controle foi objeto de consideração por um comitê de peritos da OMS (1). No continente americano 200 milhões de pessoas estão poliparasitadas, a maioria apenas infectada. Em 5 a 15\% da população observou-se a presença de sintomas devido a helmintos intestinais; cerca de

\footnotetext{
Universidade Federal de Uberlândia. Endereço: Av. Pará, 1720 Campus Umuarama, CEP 38400902, Uberlândia, Minas Gerais, Brasil. Correspondência e pedidos de separatas devem ser enviados para Carlos Henrique A. de Rezende.
}

20 milhões adoecem e mais de 10000 morrem ao ano por esta causa (2).

Indivíduos assintomáticos que pela natureza de seu trabalho estão em contato direto e permanente com alimentos, podem tornar-se fonte potencial de contaminação e disseminação de vários patógenos, entre eles enteroparasitas $(3,4)$. Na América Latina, enteroparasitoses em manipuladores de alimentos foram descritas no Brasil (5-7), no Chile (8-17) e no Panamá (18).

Em Uberlândia durante 1988, realizou-se um inquérito coproparasitológico em 1800 escolares de 20 estabelecimentos de ensino de primeiro grau
(19) e em 104 manipuladores de alimentos (7), que se ocupavam da merenda escolar naquelas escolas. $\mathrm{O}$ estudo demonstrou a semelhança do perfil de enteroparasitoses entre esses dois grupos, com maior freqüência dos protozoários Giardia lamblia e Entamoeba coli e maior porcentagem de infecção helmíntica por ancilostomídeos, Ascaris lumbricoides, Hymenolepis nana e Strongyloides stercoralis. A coincidência observada corrobora a antecipada relação, entre a transmissão de enteroparasitas e as condições de vida da população; neste caso, tanto manipuladores de alimentos quanto alunos 
viviam em condições semelhantes, com domicílio próximo à escola.

A importância dos manipuladores de merenda escolar como potenciais transmissores de enteroparasitoses e a possibilidade de interromper este elo na cadeia de transmissão, motivou os autores a realizar o presente estudo. $\mathrm{O}$ objetivo foi o de delinear o perfil das enteroparasitoses entre os manipuladores de alimento das escolas públicas de primeiro grau da área urbana do município de Uberlândia, com acompanhamento prospectivo e registro de possíveis mudanças do referido perfil após tratamento específico.

\section{MATERIAIS E MÉTODOS}

Uberlândia está situada na região do Triângulo Mineiro (figura 1) no entroncamento de estradas que ligam o Brasil no sentido norte-sul (Belém-São Paulo) e leste-oeste (Vitória-Porto Velho), com uma população total de 366711 habitantes, segundo o censo de 1991, distribuídos numa área de $4040 \mathrm{~km}^{2}$ (20). Esta configuração geográfica confere ao município grande privilégio sob o ponto de vista do desenvolvimento sócio-econômico. A partir do Programa de Saúde Escolar implementado pela Universidade Federal de Uberlândia em 1979, surgiram em 1981 as Ações Integradas de Saúde Escolar; com uma abordagem multidisciplinar, estabeleceu-se um convênio entre a Universidade Federal de Uberlândia (UFU), a Fundação de Assistência ao Estudante, a 260 Delegacia Regional de Ensino, a Diretoria Regional de Saúde e a Secretaria de Saúde da Prefeitura Municipal de Uberlândia. A alimentação escolar, fornecida pelo Programa Estadual de Alimentação Escolar, consistia de refeições preparadas em cantinas das próprias escolas, que empregavam cantineiras e outros profissionais auxiliares.

O acompanhamento prospectivo de 264 manipuladores de alimentos de 57 escolas públicas de ensino de primeiro grau da área urbana de Uberlândia, constou da realização de três exames parasitológicos de fezes. Entre os par-
FIGURA 1. Localização da cidade de Uberlândia (Minas Gerais), Brasil

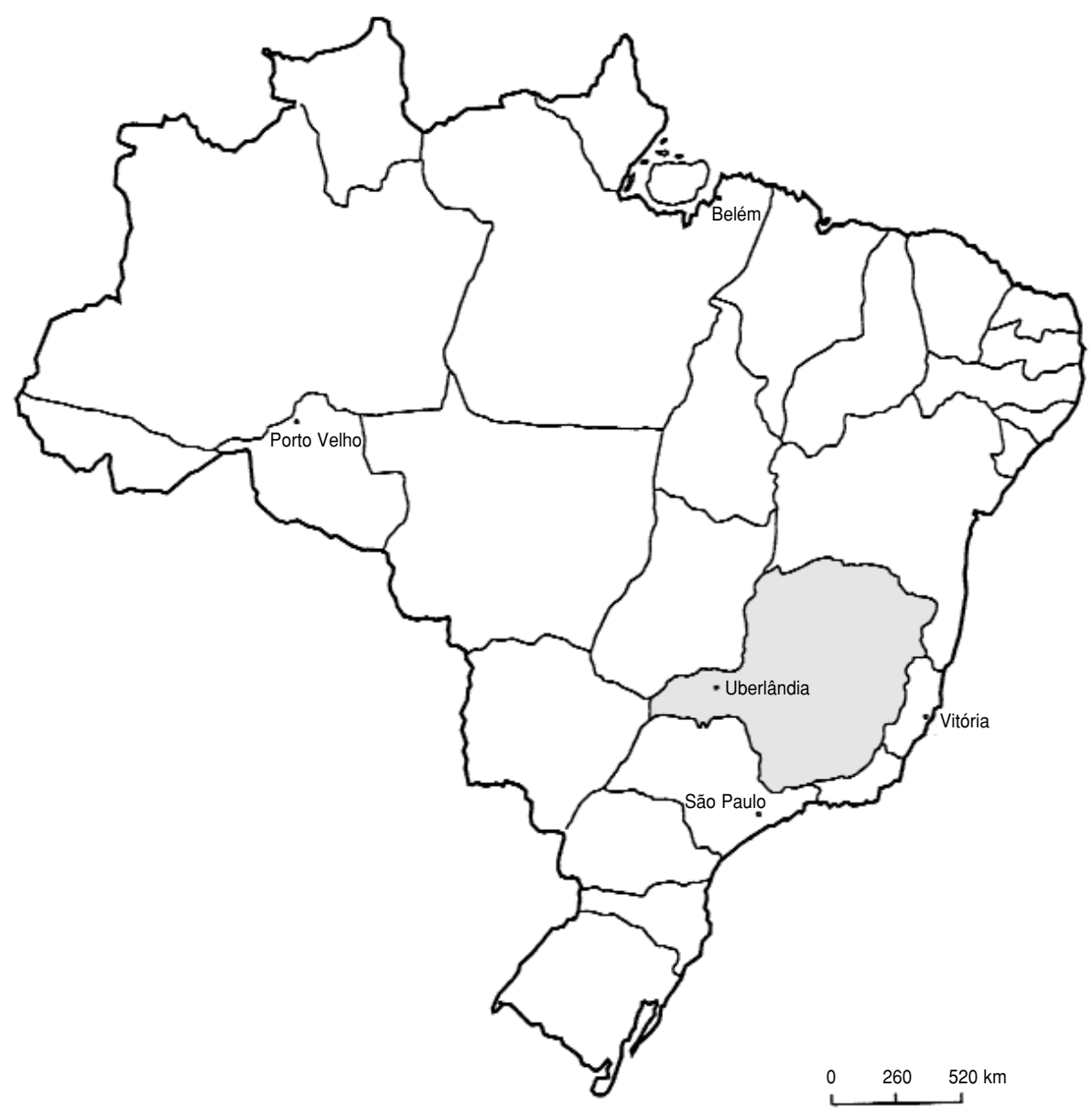

ticipantes, 259 eram do sexo feminino e 5 do sexo masculino, com idades entre 20 e 66 anos. O critério de inclusão dos indivíduos foi o consentimento para realização da pesquisa e a participação com material fecal nas três coletas (C1 em setembro de 1989, C2 em abril de 1990 e C3 em setembro de 1990). Os participantes deste estudo representavam $75 \%$ do total de 460 pessoas com que se iniciaram os estudos nas 59 escolas públicas da área urbana de Uberlândia.

Para cada indivíduo preencheu-se uma ficha de identificação com o número de ordem mantido nas três amostras, o nome da escola e do funcionário, seu endereço, bairro, função na escola, sexo, idade e a data da coleta. A ficha continha, ademais, uma pergunta sobre o conhecimento do funcionário a respeito da transmissão de parasitoses. Todos os participantes, instruídos sobre a forma de coleta e acondicionamento do material fecal, recebiam um frasco plástico com formol a $10 \%$, para cada amostra $(\mathrm{C} 1, \mathrm{C} 2$, C3). As amostras, coletadas nos domicílios e encaminhadas às escolas para recolhimento, obedeceram o intervalo programado de uma semana entre cada coleta.

A análise das 792 amostras de aproximadamente $10 \mathrm{~g}$ de fezes, feita no Laboratório de Parasitologia do Centro de Ciências Biomédicas da Universidade Federal de Uberlândia, utilizou o método Hoffman, Pons e Janer (21), com leitura microscópica do sedimento, corado com lugol, em tripli- 
cata. Empregou-se aproximadamente $4 \mathrm{~g}$ de fezes colocadas em um Becker com cerca de $5 \mathrm{ml}$ de água e trituradas com bastão de vidro. Acrescentou-se $20 \mathrm{ml}$ de água e filtrou-se a suspensão para um cálice cônico de $200 \mathrm{ml}$ por meio de gaze cirúrgica dobrada em quatro, colocada sobre tela metálica com cerca de 80 a 100 malha por $\mathrm{cm}^{2}$. Os detritos contidos na gaze, adicionados a $20 \mathrm{ml}$ de água, depois de agitados com o bastão de vidro, formavam uma suspensão, que permaneceu em repouso à temperatura ambiente por 24 horas. Após este período, descartou-se o líquido sobrenadante, quando turvo, sem perder o sedimento, o qual foi ressuspendido em água até completar $200 \mathrm{ml}$, deixado em repouso por mais duas horas. Quando o líquido estivesse límpido procedeu-se à leitura. Desprezava-se o sobrenadante e com o auxilio de uma pipeta retiravase o material para o exame microscópico. Com o sedimento colocado sobre uma lâmina, adicionado de uma gota de lugol, homogeneizado e coberto por uma lamínula de $24 \times 24 \mathrm{~mm}$, procedeu-se ao exame com aumento de 10 e 40 vezes em microscópio óptico Olympus CH2.

Todos os indivíduos receberam os resultados das três amostras, 45 dias após cada coleta. Aqueles cujos resultados demonstraram comensais (E. coli e Endolimax nana) não foram considerados positivos, uma vez que os indivíduos não receberiam tratamento específico. Os indivíduos parasitados receberam tratamento específico, por via oral, segundo a etiologia do parasita: S. stercoralis - tiabendazol 50 $\mathrm{mg} / \mathrm{kg}$ até um total de $3 \mathrm{~g}$, dividido em 2 a 3 doses ao dia; outros helmintos - mebendazol 100 mg, 2 vezes ao dia, durante 3 dias, com exceção da Taenia, cuja dose foi dobrada para $400 \mathrm{mg} /$ dia durante 3 dias; G. lamblia e E. histolytica - metronidazol $40 \mathrm{mg} / \mathrm{kg} / \mathrm{dia} \mathrm{du}$ rante 7 dias.

\section{RESULTADOS}

Entre as 264 pessoas estudadas, 67 (25\%) contratadas para exercer a função de cantineira, e 197 (75\%) para as funções de servente, serviçal e zelador, todas manipulavam alimentos por ocasião do preparo das refeições para os alunos. Todos indivíduos demonstraram conhecimento sobre transmissão de parasitoses. A ocorrência de parasitas intestinais foi de 17\% (45 casos), 10\% (27 casos) e 10\% (27 casos) para $\mathrm{C} 1, \mathrm{C} 2$ e C3, respectivamente.

A tabela 1 apresenta a distribuição por idade, sexo e ocorrência de parasitismo entre os manipuladores de alimentos registrados nas três coletas. A tabela 2 mostra a distribuição dos enteroparasitas encontrados nas três coletas realizadas de setembro de 1989 a setembro de 1990. Observa-se nesta última tabela, a identificação em $\mathrm{C} 1$, de 8 tipos de parasitas, em C2, sete tipos, e em C3, cinco parasitas diferentes. Na coleta $\mathrm{C} 1$ encontraram-se associações de parasitas, assim distribuídos: G. lamblia e ancilostomídeos (5 casos), G. lamblia e A. lumbricoides (2 casos), A. lumbricoides e T. trichiura (1 caso) e ancilostomídeos e E. histolytica (1 caso). Nas coletas C2 e C3 não se evidenciou poliparasitismo e observou-se a redução de G. lamblia, ao lado de uma constante infecção por ancilostomídeos. Embora E. coli não seja considerado um organismo patogênico, é importante salientar os índices encontrados, pois este é um parâmetro para medir o grau de contaminação fecal a que os indivíduos estão expostos. Nas amostras examinadas detectou-se este microorganismo em 42 casos (16\%) em C1, em 36 casos (14\%) em C2 e em 72 casos (27\%) em C3.

A tabela 3 apresenta os resultados segundo cada coleta, quanto ao estado de portador de helmintos e protozoários parasitas entre os manipuladores de alimentos estudados.

Entre os cinco manipuladores que apenas negativaram a amostra em C3, quatro apresentaram os mesmos para-

TABELA 1. Distribuição da ocorrência de enteroparasitoses por idade e sexo, entre manipuladores de alimentos de 57 escolas públicas de primeiro grau de Uberlândia (Minas Gerais), Brasil, 1989-1990

\begin{tabular}{|c|c|c|c|c|c|c|c|c|c|c|c|c|c|c|c|c|c|c|c|c|}
\hline \multirow{3}{*}{$\begin{array}{l}\text { Idade } \\
\text { (anos) }\end{array}$} & \multirow{2}{*}{\multicolumn{2}{|c|}{ Examinados }} & \multicolumn{6}{|c|}{$C 1^{a}$} & \multicolumn{6}{|c|}{$\mathrm{C} 2^{\mathrm{b}}$} & \multicolumn{6}{|c|}{$\mathrm{C} 3^{\mathrm{c}}$} \\
\hline & & & \multicolumn{2}{|c|}{ M } & \multicolumn{2}{|c|}{$\mathrm{H}$} & \multicolumn{2}{|c|}{ Total } & \multicolumn{2}{|c|}{ M } & \multicolumn{2}{|c|}{$\mathrm{H}$} & \multicolumn{2}{|c|}{ Total } & \multicolumn{2}{|c|}{ M } & \multicolumn{2}{|c|}{$\mathrm{H}$} & \multicolumn{2}{|c|}{ Total } \\
\hline & No. & $\%$ & + & - & + & - & No.d & $\%^{e}$ & + & - & + & - & No. & $\%$ & + & - & + & - & No. & $\%$ \\
\hline 20-29 & 8 & 3 & 3 & 3 & 1 & 1 & 4 & 50 & 0 & 6 & 1 & 1 & 1 & 13 & 0 & 6 & 0 & 2 & 0 & 0 \\
\hline 30-39 & 86 & 33 & 21 & 64 & 0 & 1 & 21 & 24 & 13 & 72 & 0 & 1 & 13 & 15 & 10 & 75 & 0 & 1 & 10 & 12 \\
\hline $40-49$ & 117 & 44 & 13 & 104 & 0 & 0 & 13 & 11 & 10 & 107 & 0 & 0 & 10 & 9 & 12 & 105 & 0 & 0 & 12 & 10 \\
\hline $50-59$ & 44 & 17 & 5 & 37 & 0 & 2 & 5 & 11 & 2 & 40 & 0 & 2 & 2 & 5 & 4 & 38 & 0 & 2 & 4 & 9 \\
\hline $60-69$ & 9 & 3 & 2 & 7 & 0 & 0 & 2 & 22 & 1 & 8 & 0 & 0 & 1 & 11 & 1 & 8 & 0 & 0 & 1 & 11 \\
\hline Total & 264 & 100 & 44 & 215 & 1 & 4 & 45 & 17 & 26 & 233 & 1 & 4 & 27 & 10 & 27 & 232 & 0 & 5 & 27 & 10 \\
\hline
\end{tabular}

$\mathrm{M}=$ mulheres; $\mathrm{H}=$ homens; + = positivo; - = negativo.

a Coleta em setembro de 1989.

${ }^{b}$ Coleta em abril de 1990.

c Coleta em setembro de 1990

¿ Número de indivíduos parasitados.

e Porcentagem de indivíduos parasitados por idade. 
TABELA 2. Distribuição de enteroparasitas em manipuladores de alimentos de 57 escolas públicas de primeiro grau de Uberlândia (Minas Gerais), Brasil, 1989 a 1990

\begin{tabular}{|c|c|c|c|c|c|c|}
\hline \multirow[b]{2}{*}{ Enteroparasitas } & \multicolumn{2}{|c|}{$\mathrm{C} 1^{\mathrm{a}}$} & \multicolumn{2}{|c|}{$\mathrm{C} 2^{\mathrm{b}}$} & \multicolumn{2}{|c|}{$\mathrm{C} 3^{\mathrm{C}}$} \\
\hline & No. & $\%^{d}$ & No. & $\%$ & No. & $\%$ \\
\hline \multicolumn{7}{|l|}{ Helmintos } \\
\hline Ancilostomídeos & 15 & 6 & 14 & 5 & 12 & 5 \\
\hline Ascaris lumbricoides & 8 & 3 & 3 & 1 & 6 & 2 \\
\hline Strongyloides stercoralis & 2 & 1 & 1 & 0 & 1 & 0 \\
\hline Hymenolepis nana & 1 & 0 & 2 & 1 & 0 & 0 \\
\hline Taenia sp. & 1 & 0 & 1 & 0 & 0 & 0 \\
\hline Trichuris trichiura & 1 & 0 & 0 & 0 & 0 & 0 \\
\hline Enterobius vermicularis & 0 & 0 & 2 & 1 & 1 & 0 \\
\hline Subtotal & 28 & 11 & 23 & 9 & 20 & 8 \\
\hline \multicolumn{7}{|l|}{ Protozoários } \\
\hline Giardia lamblia & 22 & 8 & 5 & 2 & 8 & 3 \\
\hline Entamoeba histolytica & 4 & 2 & 0 & 0 & 0 & 0 \\
\hline Subtotal & 26 & 10 & 5 & 2 & 8 & 3 \\
\hline Total & 54 & - & 28 & - & 28 & - \\
\hline
\end{tabular}

a Coleta em setembro de 1989.

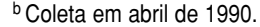

${ }^{c}$ Coleta em setembro de 1990

d Porcentagem de ocorrência do parasita em relação às 264 amostras coletadas.

TABELA 3. Estado de portador de enteroparasitas, protozoários e helmintos dos manipuladores de alimentos, segundo a coleta, em 57 escolas públicas de primeiro grau de Uberlândia (Minas Gerais), Brasil, 1989 a 1990

\begin{tabular}{cccccc}
\hline & \multicolumn{3}{c}{ Coleta } & & \multicolumn{2}{c}{ Manipuladores de alimentos } \\
\cline { 2 - 5 } $\mathrm{C} 1^{\mathrm{a}}$ & $\mathrm{C}^{\mathrm{b}}$ & $\mathrm{C}^{\mathrm{c}}$ & & No. & $\%$ \\
\hline- & - & - & 35 & 68 \\
+ & - & - & 20 & 8 \\
- & - & + & 18 & 7 \\
- & + & - & 5 & 2 \\
+ & + & - & 3 & 1 \\
+ & - & + & 2 & 1 \\
- & + & + & 2 & 1 \\
+ & + & + & 264 & - \\
\hline
\end{tabular}

= negativo; + = positivo.

a Coleta em setembro de 1989

${ }^{\mathrm{b}}$ Coleta em abril de 1990.

${ }^{c}$ Coleta em setembro de 1990

sitas nas duas primeiras amostras: em dois, detectou-se G. lamblia, em um, ovos de ancilostomídeos; em outro, ovos de Taenia sp. Somente em um caso houve detecção de parasitas diferentes: em C1 E. histolytica, e em C2 A. lumbricoides. Entre os três manipuladores que tiveram as amostras C1 e C3 positivas, os parasitas estavam assim distribuídos, por ordem de coleta: $G$. lamblia, negativo, ancilostomídeo; G. lamblia, negativo, G. lamblia e A. lumbricoides juntamente com $T$. trichiura, negativo e A. lumbricoides. Entre os dois indivíduos que tiveram somente $\mathrm{C} 2 \mathrm{e}$ C3 positivos a distribuição foi de: $G$. lamblia e G. lamblia e ancilostomídeos e A. lumbricoides. Somente em dois casos o resultado foi positivo nas três coletas, um apresentou G. lamblia, G. lamblia e ancilostomídeos, outro, S. stercoralis, ancilostomídeos e A. lumbricoides.

\section{DISCUSSÃO}

Em todos os programas nacionais de alimentação infantil ou de alimentação e nutrição, deveriam integrar-se componentes educativos, baseados na análise de risco potencial de contaminação dos alimentos e na identificação de pontos críticos de controle, considerados sempre os fatores sócio-culturais (22).

Neste estudo, 75\% dos indivíduos que manipulavam alimentos nas escolas exerciam funções de serventes, serviçais e zeladores cujas atividades incluíam a limpeza dos sanitários, o que poderia ser um fator de risco. A propósito, já foi demonstrada a contaminação dos sanitários em escolas por ovos de helmintos com freqüência decrescente em: assentos, trincos, maçanetas internas e botões de descarga (23). A freqüência de ovos de enteroparasitas nos sanitários de uso público, embora não seja parâmetro para avaliar o índice de contaminação dos usuários, indica a presença dos agentes infecciosos nesses locais.

Em 1988, demonstrou-se a ocorrência de parasitas intestinais (inclusive $E$. coli) em $47 \%$ de um total de 104 manipuladores de merenda escolar em 20 estabelecimentos de ensino público de primeiro grau da área urbana de Uberlândia, pela análise de uma única amostra de fezes (7). No presente trabalho com a extensão da amostra de 20 para 57 das 59 escolas públicas da mesma área, detectou-se $17 \%$ de positividade em $\mathrm{C} 1$, excluída a infecção por E. coli. Por outro lado, a cobertura de população neste inquérito foi muito ampla, incluía indivíduos com três amostras de fezes, com seguimento por meio de controle semestral, que correspondia a $75 \%$ do total de manipuladores de alimentos das escolas.

Na população estudada, obtiveramse nos três semestres, resultados inferiores aos de Frenzel et al. em 1979 (11) na cidade de Valdivia, Chile, quando estudaram parasitoses intestinais em manipuladores de alimentos para lactantes, obtiveram 34\% de infecção, sem considerar os parasitas não patogênicos e $54 \%$ de positividade quando incluíam os comensais. 
Verifica-se na tabela 1 que, embora a faixa etária de 20 a 29 anos seja a de menor número de funcionários é a de maior positividade, seguida da faixa etária de 30 a 39 anos, voltando a elevar a taxa nos indivíduos de mais idade. Com o tratamento específico após os resultados de $\mathrm{C} 1$, observou-se decréscimo da positividade em todas as faixas etárias em C2 e ausência de parasitas na faixa etária de 20 a 29 anos em C3. Embora nestes intervalos de tempo possa ter havido reinfecção, observouse diminuição do grau de parasitismo em relação à amostra inicial.

Tal como no inquérito anterior (7), embora em porcentagem menor, $\mathrm{O}$ presente trabalho em C1 (tabela 2) detectou os mesmos quatro helmintos mais freqüentes: ancilostomídeos, $A$. lumbricoides, S. stercoralis e H. nana, e a presença de mais três parasitas: Taenia sp., T. trichiura e E. vermicularis, anteriormente não detectados. Como o método utilizado foi o mesmo (21), e não específico para detecção de ovos de Taenia sp. e E. vermicularis, suspeita-se que a infecção seja ainda mais elevada. O mesmo poderia ter acontecido com a positividade para $S$. stercoralis, se o método de detecção pelo termotropismo das larvas tivesse sido utilizado. Entre os helmintos a predominância de ovos de ancilostomídeos continua chamando a atenção desde a publicação de Costa-Cruz et al. em 1995 (7). Na América Latina, essa parasitose só é relatada em manipuladores de alimentos no Brasil; os trabalhos do Chile (8-17) e do Panamá (18) não mencionaram esta infecção nestes tra- balhadores. Ao tempo da terceira coleta de amostras observou-se (tabela 2) a negativação para $H$. nana, Taenia sp. e T. trichiura. Devido à transmissão predominantemente indireta, a reinfecção pelos helmintos pode ser facilitada apesar do tratamento específico.

Quanto aos protozoários, G. lamblia foi o parasita mais freqüentemente detectado na primeira coleta (22 casos, $8 \%$ ) se comparado ao resultado de trabalho anterior. Torres et al. em 1991 (6), utilizaram a mesma metodologia do presente trabalho e relataram a ocorrência de $4 \%$ de giardíase entre 50 manipuladores de alimentos de 18 creches, mantidas pela Prefeitura do Município de São Paulo, durante os anos de 1982 e 1983. A ocorrência de $E$. histolytica passou de 3\% em 1988 (7) para $2 \%$ em $C 1$, e não foi detectada nas coletas subseqüentes. As condições precárias de manipulação de alimentos (falta e/ou inadequação de utensílios, destinação incorreta do lixo), possibilitaria a manutenção de ciclos de infecção principalmente ligados aos protozoários. Assim sendo, o tratamento específico com metronidazol teria uma importância limitada. A modificação observada na freqüência destes dois protozoários entre C1, C2 e C3 pode relacionar-se à limpeza de caixas d'água nas escolas, variável não prevista antes da conclusão deste estudo. A detecção dos mesmos parasitas em duas amostras consecutivas C1 e C2 ou C2 e C3 pode representar falha terapêutica ou reinfecção.

Verifica-se na tabela 3 que os 45 casos positivos em $\mathrm{C} 1$ diminuíram para 27 nas outras duas coletas embora em circunstâncias diferentes, uma vez que em C3 foram detectados pela primeira vez 20 casos de parasitismo. Esta situação alerta para a necessidade da implantação de vigilância epidemiológica por meio de exames parasitológicos periódicos e tratamento específico. Considera-se igualmente necessário que todos os manipuladores de alimentos dos estabelecimentos de ensino, tenham condições adequadas de trabalho e recebam treinamento sobre manejo de alimentos e higiene pessoal.

\section{AGRADECIMENTO}

Este estudo teve o patrocínio do Programa de Ações Integradas de Saúde Escolar, por meio de convênio entre a Universidade Federal de Uberlândia, a Secretaria Municipal de Saúde, a Diretoria Regional de Saúde e a 26 $6^{\text {a Delega- }}$ cia Regional de Ensino.

Agradecemos ao Geraldo O. Rodrigues da Motta e à Maria das Graças Marçal pelo apoio técnico laboratorial, à equipe do Programa de Ações Integradas de Saúde Escolar do Município de Uberlândia pela colaboração ao professor Uriel Franco Rocha pelas sugestões à redação deste trabalho e a professora Marlene Terezinha Muno Colesanti pelo fornecimento do mapa do Brasil produzido no laboratório de geoprocesamento do Departamento de Geografia da Universidade Federal de Uberlândia

\section{REFERÊNCIAS}

1. Organización Mundial de la Salud. Prevención y control de infecciones parasitarias intestinales: informe de un Comité de Expertos de la OMS. Ginebra: OMS; 1987:87. (Informe técnico 749).

2. Apt W. Helmintiasis intestinales humanas en América Latina: prevalencia actual y sus factores contribuyentes. Parasitol Dia (Santiago, Chile) 1987;11:155-166.

3. Jackson GJ. Public health and research perspectives on the microbial contamination of foods. J Anim Sci 1990;68:884-891.

4. Quick R, Paugh K, Addiss D, Kobayashi J, Baron R. Restaurant-associated outbreak of giardiasis. J Infect Dis 1992;166:673-676.
5. Munhoz RAR, Faintuch MB, Valtorta A. Enteroparasitoses em pessoal de nutrição de um hospital geral: incidência e valor da repetição dos exames. Rev Hosp Clin Fac Med Sao Paulo 1990;45:57-60.

6. Torres DMAGV, Chieffi PP, Costa WA, Kudzielics E. Giardíase em creches mantidas pela Prefeitura do Município de São Paulo, 1982/1983. Rev Inst Med Trop Sao Paulo 1991; 33:137-142

7. Costa-Cruz JM, Cardoso MLG, Marques DE. Intestinal parasites in school food handlers in the city of Uberlândia, Minas Gerais, Brazil. Rev Inst Med Trop Sao Paulo 1995;37:191-196.
8. Reyes H, Olea M, Hernández R. Enteroparasitosis en manipuladores de alimentos del Área de Salud Oriente de Santiago. Bol Chil Parasitol 1972;27:115-116.

9. Dall'orso LM, Pinilla N, Parra G, Bull F. Parásitos y protozoos comensales intestinales en manipuladores de alimentos del área central de la ciudad de Concepción, Chile. Bol Chil Parasitol 1975;30:30-31.

10. Reyes $M H$, Muñoz V. Enteroparasitosis en manipuladores de alimentos en hospitales. Rev Med Chil 1975;103:477-479.

11. Frenzel A, Torres P, Guerrero S, Geshe W, Montefusco A, Marín P. Parasitosis intestinal 
en lactantes y su relación con la infección de sus manipuladores de alimentos y el saneamiento ambiental. Rev Med Chil 1979;107: 343-351.

12. Díaz MC, Muñoz V, Durruty J, Osorio M. Estudio bacteriológico y parasitológico en manipuladores de alimentos de servicios hospitalarios. Rev Med Chil 1980;108:900-902.

13. Fuentes A, Sagua H, Araya R, González J. Estudio parasitológico de manipuladores de alimentos en la ciudad de Antofagasta. Rev Chil Tecnol Med 1981-82;IV(2):69-71.

14. Krstulovic L, Reyes V. Enteroparasitosis en manipuladores de alimentos de la Comuna de El Monte. Parasitol Dia (Santiago, Chile) 1984; 8:13-14.

15. Lobato Il, Arriagada JP, Núñez JO, Regente PI, Maldonado MC. Enteroparasitosis en manipuladores de alimentos de la ciudad de Arica, I Región, Chile. Rev Chil Tecnol Med 1985;8:355-356.
16. Arias B, Soto E, Sepúlveda L, Herrera A. Infección intestinal por parásitos o comensales en manipuladores de alimentos de hospitales del sector norte de Santiago, Chile. Bol Chil Parasitol 1987;42:84-86.

17. Henríquez MAB, Castelblanco CG. Enteroparasitosis en manipuladores de alimentos. Rev Chil Nutr 1989;17:122-125.

18. Sánchez JL, Ríos C, Hernández-Fragoso I, Ho CK. Parasitological evaluation of a food handler population cohort in Panama: risk factors for intestinal parasitism. Milit Med 1990; 155:250-255.

19. Costa-Cruz JM, Ambrosio MR, Marques DE, Gennari-Cardoso ML, Couto JDV. Inquérito coproparasitológico em escolares de Uberlândia, MG. Rev Soc Bras Med Trop 1991;24:(Supl II): 141 .

20. Instituto Brasileiro de Geografia e Estatística. Enciclopédia dos municipios brasileiros. Rio de Janeiro, Brasil: IBGE 1959; 1527: 400-405.
21. Hoffman WA, Pons JA, Janer JL. The sedimentation concentration method in schistosomiasis mansoni. Puerto Rico J Publ Health Trop Med 1934;9:283-298.

22. Motajermi Y, Käferstein F, Moy G, Quevedo F. Alimentos de destete contaminados: um importante factor de riesgo de diarrea y malnutrición asociada. Bol Oficina Sanit Panam 1994;116:313-330.

23. Aidar-Sobrinho T, Coelho LMPS, Oliveira SM, Martins JT, Rabello Júnior JA, Oliveira CRP, et al. Estudo da freqüência de ovos de helmintos intestinais em sanitários de uso público de Sorocaba, SP. Rev Soc Bras Med Trop 1995; 28:33-37.

Manuscrito recebido em 16 de junho de 1995. Aceito em versão revisada em 3 de maio de 1996.

ABSTRACT A prospective study based on a parasitologic survey was carried out with a view toward controlling enteroparasitoses in food handlers who work in public primary schools in Uberlândia, Minas Gerais, Brazil. The study subjects were 264 individuals from 57 schools, from whom three fecal samples (C) were collected: C1 in September 1989, C2 in April 1990, and C3 in September 1990. The participants were predominantly female ( 259 women and 5 men) and ranged in age from 20 to 66 years. A total of 792 samples were preserved in $10 \%$ formalin and analyzed using the method of Hoffman, Pons, and Janer. Intestinal parasites were found in $17 \%, 10 \%$, and $10 \%$ of the $\mathrm{C} 1, \mathrm{C} 2$, and $\mathrm{C} 3$ samples, respectively. The following organisms were found in $\mathrm{C} 1$ : Giardia lamblia (8\%), ancylostomids (6\%), Ascaris lumbricoides (3\%), Entamoeba histolytica (2\%), Strongyloides stercoralis, Hymenolepis nana, Taenia sp., and Trichuris trichiura (less than 1\% each). Parasites found in C2 were: ancylostomids (5\%), G. lamblia (2\%), A. lumbricoides (1\%), S. stercoralis, H. nana, Taenia sp., and Enterobius vermicularis (less than $1 \%$ each). In C3, the parasites observed were: ancylostomids (5\%), G. lamblia (3\%), A. lumbricoides ( $2 \%)$, S. stercoralis, and E. vermicularis (less than $1 \%$ each). After each collection, the individuals found to be infected received specific treatment. The authors recommend the establishment of epidemiologic surveillance through periodic parasitologic exams, specific treatment, and training on hygienic food handling and personal hygiene for all food handlers. 